\title{
Plasma Model of Generation and Slip of Linear Defects in Crystalline Materials
}

\author{
V. L. Busov1, Grechkina Margarita Vladimirovna² \\ ${ }^{1}$ Donbass State Engineering Academy, Kramatorsk, Ukraine \\ ${ }^{2}$ Experimental Equipment Department of Voronezh State University, Voronezh, Russia \\ Email:vlbusov49@gmail.com,grechkina_m@mail.ru
}

How to cite this paper: Busov, V.L. and Vladimirovna, G.M. (2020) Plasma Model of Generation and Slip of Linear Defects in Crystalline Materials. Applied Mathematics, 11, 1167-1177.

https://doi.org/10.4236/am.2020.1111079

Received: June 22, 2020

Accepted: November 17, 2020

Published: November 20, 2020

Copyright $\odot 2020$ by author(s) and Scientific Research Publishing Inc. This work is licensed under the Creative Commons Attribution International License (CC BY 4.0).

http://creativecommons.org/licenses/by/4.0/

\begin{abstract}
In dielectrics and semiconductors, a plasma model of the generation and slip of dislocations is considered, where under shock loads in a generalized space of rectangular pulses an alternating field forms a distribution of pairs of photoelectrons and cations; these electrons with velocities $V_{e}$ create $\delta$-collisions with cold plasma from free electrons and holes with masses $m_{e}$ and $m_{h}$ $\left(m_{h} \gg m_{e}\right)$, they emit and absorb longitudinal electron plasma waves whose phase velocities $\omega_{p w} / k_{p w}$ are close to or are equal to the velocities $V_{e}$, while the frequencies $\omega_{p w}$ and wave numbers $k_{p w}$ of the wave packet of plasma waves are complex, the short-wave components $\left(k_{p w}^{-1}\right)_{\min }$ of this wave packet at $k_{p w} \cdot a_{e} \gg 1$ ( $a_{e}$-Debye screening radius) decay in the core linear defect, and its long-wavelength components $\left(k_{p w}^{-1}\right)_{\max }$ propagate in the region of the medium surrounding the core of the defect at $k_{p w} \cdot a_{e}<\cong 1$. When a defect is generated, the distribution of cations under the influence of the internal Coulomb field shifts to the region of the first peak (protrusion) of the electron plasma wave, thereby forming a vacancy valley. When sliding under the influence of an external electric field, a cationic plasma wave consisting of a vacancy valley and two cationic protrusions moves against the background of an additional potential relief created by an electron plasma wave near the core of the defect. It has been shown that $\delta$-collisions create flows of dynamic large-scale correlations of plasma fluctuations in the form of asymptotics of different-time correlators of density and potential fluctuations as $t \rightarrow+\infty$.
\end{abstract}

\section{Keywords}

Solid-State Plasma, Debye Screening Radius, Charged Particle Collisions, Plasma Wave, Flows of Dynamic Correlations of Density, Potential Fluctuations 


\section{Introduction}

At present, the fundamental theory of the nuclei of linear defects in semiconductors is the Reed discrete static model [1] [2], where it was assumed that an additional half-plane of the edge dislocation introduces a chain of free or unsaturated bonds into the crystal. Due to the attraction of free electrons to these bonds in germanium, as well as in most cases, a negative linear charge of a dislocation core arises in silicon, which forms a cylindrical space charge in the environment surrounding the core with a radius $R$ from 1 to $10 \mu \mathrm{m}$. Using the Poisson equation and the electrostatic energy of trapped electrons, Reed found the work of space charge formation for one additional electron in the chain

$$
A_{p r}=f \cdot E_{0}\left(\frac{3}{2} \ln \frac{f}{f_{c}}-0.866\right)
$$

where $f$ is the degree of filling of the chain is determined by the Fermi distribution function

$$
f=\left[1+\exp \frac{\left(E_{d}-E_{f}\right)}{k T}\right]^{-1}
$$

where $E_{d}$ is the dislocation level within the band gap; $E_{f}$ is the Fermi level; $T$ is the absolute temperature; $E_{0}$ is the interaction energy of neighboring electrons in the chain, $E_{0}=e^{2} / \epsilon a_{c h} ; a_{c h}$ is the distance between neighboring trapped electrons; $1 / a_{c h}$ is the density of additional electrons per unit length of the dislocation; $\epsilon$-dielectric constant; $f_{c}$ is distribution function $f=f_{c}$ at $R=a_{c h} ; c$ is the distance between adjacent unsaturated bonds, the ratio $a_{c h} / c$ is of the order of 0.1. Reed also found the potential of the field $\phi_{0}$ created by all other electrons of the linear charge and positive space charge at the location of an arbitrary injected electron

$$
\phi_{0}=\frac{e}{\epsilon c} f\left(3 \ln \frac{f}{f_{c}}-1.232\right)
$$

The numerical value of was confirmed experimentally by scanning with an electron beam except for its sign ([1], p. 112). Of particular interest is the dependence of the electrostatic potential of the barrier $\phi_{b}$ on the distance $r$ from the dislocation line ([1], Figure 8.25, p. 178), where $\phi_{b}(r)$ obtained at an average concentration of conduction electrons $n_{e s}=1 \times 10^{15} \mathrm{~cm}^{-3} ; a_{c h}=2.85 \mathrm{~nm}$; $c=0.4 \mathrm{~nm} ; c / a_{c h}=0.14 ; E_{d}=0.4 \mathrm{eV}$ below the boundary of the conduction band, and consists of two branches: ascending at $r=0$ from $0.18 \mathrm{~V}$ to the maximum of the curve $0.27 \mathrm{~V}$ at $r_{m}=6 \div 10 \mathrm{~nm}$ and descending from $r_{m}$ to $r_{f} \approx 100 \mathrm{~nm}$. It should be noted that the distance $r_{m}$ is associated with the wavelength $\lambda_{i e}$ of the injected electron

$$
r_{m}=\frac{e^{2}}{2 \pi \epsilon k T} \ln \frac{R}{\lambda_{i e}}
$$

Here the question arises: How does the generation and slip of linear defect 
nuclei occur in the Reed model?

On the other hand, in the limiting case, near the electric breakdown and shock compression of crystalline dielectrics and semiconductors, there occurs a solid-plasma phase transition [3], where the combined influence of electric fields is $E^{\text {out }}=10^{9}-10^{10} \mathrm{~V} / \mathrm{m}$ and hydrostatic pressures $p=10^{9}-10^{11} \mathrm{~Pa}$ lead to two effects: 1) reduction of the band gap $E_{g}^{*}(p)$ according to the linear law

$$
E_{g}^{*}(p)=E_{g}^{0}(p)+a_{p} \cdot p
$$

where $a_{p}$ is the baric coefficient expressed in terms of the compressibility coefficient $K$ and has a negative value

$$
a_{p}=\gamma \cdot K
$$

where $9 \times 10^{-12} \leq K \leq 200 \times 10^{-12} ; \gamma \cong-(2 \div 3) \times 10^{-19} \mathrm{~J}$ the generation of electron-hole pairs, the probability $w$ of occurrence of which has the form [4] [5]

$$
w=n_{e h} / N=\frac{\left(e E^{\text {out }} \cdot a_{0}^{*}\right)^{2}}{2 \pi \hbar \cdot E_{g}^{*}} \exp \left(-\frac{E_{g}^{*}}{e a_{0}^{*} \cdot E^{\text {out }}} \ln 1 / \alpha\right)
$$

where $n_{e h}$ and $N$ are the average densities of electron-hole pairs formed per unit time and valence electrons, respectively; $a_{0}^{*}$ is the effective lattice parameter; $\alpha$ is the ratio of the width of the valence band to the width of the conduction band. It was shown in [3] that when injected electrons move in the volume of a shock wave in the velocity range $V_{i e}=4 \times 10^{3} \div 1 \times 10^{6} \mathrm{~m} / \mathrm{s}$ and pressures $p=1.2 \times 10^{10} \div 1 \times 10^{11} \mathrm{~Pa}$ for alkali halide crystals of the $\mathrm{NaCl}$ type, the value of $E_{g}^{*}(p)$ decreases from 8.6 to $1.5 \mathrm{eV}$.

It is known [6] that the energy of the ground state of a solid-state plasma is mainly determined by electrostatic energy, and as a second-order correction it contains the correlation energy $W$, which is divided into two components: $W_{1}$, due to large-scale Coulomb correlations in the relative motion of electrons, and $W_{s}$, due to the Pauli principle in the form of small-scale correlations with an effective radius of $0.1-0.2 \mathrm{~nm}$. An analysis of the works [7] [8], which consider the processes of damage formation in ionic crystals under the influence of intense laser radiation, shows that here the decisive role is played by the influence of plasma arising both at the boundaries of blocks, grains, cleavage, and in the volumes of pores and cavities.

The well-known Reed model does not reflect the combined dynamics of conduction electrons captured by unsaturated bonds and the atomic collective near the dislocation line during generation and slip of the dislocation core. The question naturally arises: How is the generation and sliding of linear defects in a solid-state plasma under impact loads?

The aim of this work is to build a plasma model of the processes of generation and slip of linear defects in dielectrics and semiconductors.

\section{The Theoretical Model}

We find the Debye radius of screening of a solid-state plasma ([9], p. 145) 


$$
a_{e}=\left\{\frac{k T}{4 \pi n_{e} \cdot e^{2}}\right\}^{1 / 2}
$$

where $e$ is the electron charge; $n_{e} \equiv n_{e m}, n_{e s}, n_{e d}$ are the average electron densities in metals, semiconductors, and dielectrics, respectively. At room temperature and $n_{e m}=1 \times 10^{22}-6 \times 10^{23} \mathrm{~cm}^{-3} \quad([10]$, p. 380$)$ the $a_{e m}$ radius in metals is $25 \div 30 \mathrm{~nm}$, at low temperatures $4-6^{\circ} \mathrm{K} a_{e m}=4-5 \mathrm{~nm}$. In semiconductors doped with impurity atoms, $n_{e s} \approx 10^{16}-10^{18} \mathrm{~cm}^{-3}$, the radius $a_{e s}$, according to (8), takes values from several $\mathrm{mm}$ to several $\mathrm{cm}$. In pure Ge, $n_{e s} \approx 10^{13}-10^{14} \mathrm{~cm}^{-3}$ is comparable to or slightly larger than $n_{e d}$ alkali halide crystals.

At the first stage of the theory, we assume that under the influence of impurity ions, external electric fields and shock waves, the material already has an equilibrium neutral distribution of free electrons and holes with masses $m_{e}$ and $m_{h}$ for which: 1) the effective mass of electrons is $m_{e}^{*} \ll m_{h}^{*}$-effective mass of holes; 2) the relaxation time of free electrons is $\tau_{r e} \ll \tau_{\text {rec }}$-the recombination time of the injected electrons and holes. We also emphasize that upon impact of the surface of a Ge, Si type semiconductor, an intermittent field injects valence electrons from atoms at lattice sites, turning them into cations; in the case of an insulator of the type $\mathrm{Na}^{+} \mathrm{Cl}^{-}$injection of bond electrons and the formation of light cations $\mathrm{Na}^{+}$take place.

Let us consider in the Cartesian coordinate system a certain volume $V$ of crystalline material, where an alternating field $E_{x}^{i f}$ acts in the generalized space of rectangular pulses along the $x$ axis in the standing wave regime $E_{0 x}^{i f} \delta(t)$ and $-E_{0 x}^{i f} \delta(t+\tau) \quad\left(\tau\right.$ is the pulse duration) [11] in the slip plane x0z. The field $E_{x}^{i f}$ creates in the right $(x>0)$ and left $(x<0)$ half-spaces of volume $V$ a pair of photoelectrons and cations forming two flat beams with velocities $\boldsymbol{V}_{e}$ and $-\boldsymbol{V}_{e}$, while the cation velocities $\boldsymbol{v}_{\text {cat }}$ in both half-spaces will be consider small: $v_{\text {cat }} \ll V_{e}$. Each beam in its half-space creates $\delta$-ollisions, through which it interacts with the plasma, while the chains of photoelectrons emit and absorb longitudinal plasma waves-harmonics $\exp [-i(\boldsymbol{k} \boldsymbol{r}-\omega t)]$ of only certain frequencies $\omega_{p w}$ and wave vector $\boldsymbol{k}_{p w}$, selectable using equations

$$
\begin{aligned}
\omega_{p w}-\boldsymbol{k}_{p w} \boldsymbol{V}_{e} & =0 \\
\varepsilon_{l}\left(\boldsymbol{k}_{p w}, \omega_{p w}\right) & =0
\end{aligned}
$$

where $\varepsilon_{l}\left(\boldsymbol{k}_{p w}, \omega_{p w}\right)$ is the longitudinal dielectric constant of the plasma, and obtained from the expression of the Landau collision integral ([9], p.236). In our case, we will consider the intrinsic plasma of the material to be cold: $v_{e d}, v_{e s} \ll V_{e}$, where $v_{e d}, v_{e s}$ are the average thermal velocities of plasma electrons in dielectrics (d) and semiconductors (s). Plasma electrons will be elastically scattered with respect to the direction of the beam only forward at large angles $\chi$ ([12], p. 64). The roots of the dispersion law (10) are complex $\omega_{p w}=\omega_{p w}^{\prime}+i \omega_{p w}^{\prime \prime}, \gamma=-\omega_{p w}^{\prime \prime}, \quad \gamma$ is the plasma wave attenuation decrement according to the law $\exp (-\gamma t)$ 


$$
\gamma=\sqrt{\frac{\pi}{8}} \cdot \frac{\Omega_{e}}{\left(k_{p w} a_{e}\right)^{3}} \cdot \exp \left[-\frac{1}{2\left(k_{p w} a_{e}\right)^{2}}-\frac{3}{2}\right]
$$

where $\Omega_{e}$ is the plasma frequency of electrons, $\Omega_{e}=\left\{4 \pi n_{e} e^{2} / m_{e}\right\}^{1 / 2}$; $a_{e} \equiv a_{e d}, a_{e s}$. It should be noted here that the real part $\omega_{p w}^{\prime}$ при $k_{p w} a_{e} \ll 1$

$$
\omega_{p w}^{\prime}=\Omega_{e}\left(1+\frac{3}{2}\left(k_{p w} a_{e}\right)^{2}\right)
$$

and the imaginary part

$$
\omega_{p w}^{\prime \prime}=-\frac{1}{2} \Omega_{e} \varepsilon_{l}^{\prime \prime}\left(\boldsymbol{k}_{p w}, \omega_{p w}\right)
$$

exponentially small with $\varepsilon_{l}^{\prime \prime}$. Hence, a plasma wave in the form of a long-wave harmonic is propagating if $\gamma$ is exponentially small for $k_{p w} a_{e} \ll 1$. In contrast, short-wavelength harmonics are non-propagating; they make the main contribution to the converging Balescu-Lenard collision integral $S t f_{B L}$ and essentially form the core of a linear defect. Indeed, as shown in ([9], p. 238), it is the region $k_{p w} a_{e} \gg 1$ that makes the main contribution to the integral $S t f_{B L}$. It should be noted here that the short-wave harmonics of the electron plasma wave create an internal Coulomb attraction of the cation distribution.

On the other hand, plasma waves can be considered as fluctuation waves. For times $\Delta t$ that are small compared with the period of the rectangular pulses $\omega_{\text {rep }}^{-1}$, the plasma can be considered collisionless. If, within the standard procedure, the distribution function of plasma electrons $f_{e}$ and the scalar potential $\varphi_{e}$ are divided into the regular $\bar{f}_{e}, \bar{\varphi}_{e}$ and fluctuation parts $\delta f_{e}, \delta \varphi_{e}$ : $f_{e}=\bar{f}_{e}+\delta f_{e}$ and $\varphi_{e}=\bar{\varphi}_{e}+\delta \varphi_{e}$, then the system of equations describing such a plasma has the form ([9], p. 256):

$$
\begin{gathered}
\frac{\partial \bar{f}_{e}}{\partial t}+\boldsymbol{v}_{e} \cdot \frac{\partial \bar{f}_{e}}{\partial \boldsymbol{r}}-e \frac{\partial \bar{\varphi}_{e}}{\partial \boldsymbol{r}} \frac{\partial \bar{f}_{e}}{\partial x}=e\left\langle\frac{\partial \delta \varphi_{e}}{\partial \boldsymbol{r}} \frac{\partial \delta f_{e}}{\partial \boldsymbol{p}}\right\rangle \\
\Delta \bar{\varphi}_{e}=-4 \pi e \int \bar{f}_{e} \mathrm{~d}^{3} p
\end{gathered}
$$

where the right side of (14) is the collision integral. It is clear that the terms quadratic in $\delta \varphi_{e}$ and $\delta f_{e}$ in these equations describe the effect of collisions on fluctuations. Neglecting these terms and taking $\bar{f}_{e}=\bar{f}_{e}(\boldsymbol{p}) ; \bar{\varphi}_{e}=0$, according to [9], we obtain the equations for fluctuations $\delta f_{e}$ and $\delta \varphi_{e}$ for $\Delta t \ll \omega_{\text {rep }}^{-1}$

$$
\begin{gathered}
\frac{\partial \delta f_{e}}{\partial t}+v_{e j} \cdot \frac{\partial \delta f_{e}}{\partial x_{j}}-e \frac{\partial \delta \varphi_{e}}{\partial x_{j}} \frac{\partial \overline{f_{e}}}{\partial p_{j}}=0 \\
\Delta \delta \varphi_{e}=-4 \pi e \int \delta f_{e} \mathrm{~d}^{3} p
\end{gathered}
$$

It should be noted here that $\bar{f}_{e}(\boldsymbol{p})$ reflects the nonequilibrium stationary state of a collisionless plasma, in the absence of an external field it depends only on the momenta of plasma electrons and is a stationary solution (14), (15). Equations (16) and (17) allow us to construct the asymptotics of different-time 
correlators as $t \rightarrow+\infty\left\langle\delta f_{e}\left(t_{1}, \boldsymbol{r}_{1}, \boldsymbol{p}_{1}\right) \delta f_{e}\left(t_{2}, \boldsymbol{r}_{2}, \boldsymbol{p}_{2}\right)\right\rangle$ and $\left\langle\delta f_{e}\left(t_{1}, \boldsymbol{r}_{1}, \boldsymbol{p}_{1}\right) \delta \varphi_{e}\left(t_{2}, \boldsymbol{r}_{2}, \boldsymbol{p}_{2}\right)\right\rangle$. In ([9], p. 260), Fourier images or spectral correlators of these asymptotics were found

$$
\begin{gathered}
\left(\delta \varphi^{2}\right)_{\omega \boldsymbol{k}}=\frac{32 \pi^{3} e^{2}}{k^{4}\left|\varepsilon_{l}(\omega, \boldsymbol{k})\right|^{2}} \int \bar{f}_{e}(\boldsymbol{p}) \delta\left(\omega-\boldsymbol{k} \boldsymbol{V}_{e}\right) \mathrm{d}^{3} p \\
\left(\delta \varphi_{e} \delta f_{e}\right)_{\omega \boldsymbol{k}}=\frac{e \boldsymbol{k}}{\omega-\boldsymbol{k} \boldsymbol{V}_{e}+i 0} \frac{\partial \bar{f}_{e}}{\partial \boldsymbol{p}}\left(\delta \varphi^{2}\right)_{\omega \boldsymbol{k}}+\frac{8 \pi^{2} e}{k^{2} \varepsilon_{l}(\omega, \boldsymbol{k})} \bar{f}_{e}(\boldsymbol{p}) \delta\left(\omega-\boldsymbol{k} \boldsymbol{V}_{e}\right)
\end{gathered}
$$

All three components of the bulky expression $\left(\delta f_{e} \delta f_{e}\right)_{\omega k}$ (19) also contain the product $\bar{f}_{e}(\boldsymbol{p}) \delta\left(\omega-\boldsymbol{k} \boldsymbol{V}_{\xi}\right)$, and the first $(\ldots)_{1}$-initially, and two the rest $(\ldots)_{2},(\ldots)_{3}$ - after finding $\frac{\partial f_{e}^{f}}{\partial p}$, while $(\ldots)_{1}$ represents in the momentum space a $\delta$-peak with an amplitude of $\sim n_{e}$, and $(\ldots)_{2},(\ldots)_{3}$ in $\boldsymbol{k}$-space the line spectrum with envelopes $k^{-2}$ and $k^{-1}$, respectively.

The process of generating nuclei of linear defects is divided into several stages: the formation of short-wavelength harmonics of the plasma wave $\delta f_{e}$ and their stabilization by moving the distribution of photoelectrons to the region of the first trough $\delta f_{e}$, and the distribution of cations to the region of the first peak (protrusion) $\delta f_{e}$ and the formation of a vacancy valley. Indeed, using the diffusion equations, or rather the mass transfer of cations and photoelectrons ([9], p. 118; 2, p. 157)

$$
\begin{gathered}
\frac{\partial n_{c a t}}{\partial t}=\operatorname{div}\left[-\mu_{c a t} k T \nabla_{x} n_{c a t}+n_{c a t} \mu_{c a t} z e E^{i n s}\left(\delta f_{e}(+)_{1}\right)\right] \\
\frac{\partial n_{p h e}}{\partial t}=\operatorname{div}\left[-\mu_{p h e} k T \nabla_{x} n_{p h e}+n_{p h e} \mu_{p h e} e E^{i n s}\left(\delta f_{e}(-)_{1}\right)\right]
\end{gathered}
$$

where $n_{c a t}$ and $n_{p h e}, \mu_{c a t}$ and $\mu_{p h e}$ are the average distribution densities of cations and photoelectrons and their mobility; $E^{i n s}\left(\delta f_{e}(+)_{1}\right)$ and $E^{i n s}\left(\delta f_{e}(-)_{1}\right)$ are the internal Coulomb fields caused by the distribution of plasma electrons in the regions of the first protrusion and the first trough $\delta f_{e}$, we will determine the conditions for stabilization of the defect nucleus. At $\frac{\partial n_{\text {cat }}}{\partial t}=\frac{\partial n_{\text {phe }}}{\partial t}=0$, the drift currents caused by the fields $E^{i n s}\left(\delta f_{e}(+)_{1}\right)$ and $E^{i n s}\left(\delta f_{e}(-)_{1}\right)$ are balanced by mass transfer currents from the gradients densities $n_{\text {cat }}$ and $n_{p h e}$, while the photoelectrons are redistributed to the potential valley of the first trough $\delta f_{e}$, and the cations to the potential valleys of the first protrusion $\delta f_{e}$ both on the left and on the right, provided that such stabilization time is shorter than the recombination time at the holes, and this is always true [2] [9]. In other words, the distributions of photoelectrons and cations are in quasistable states in an additional potential relief created by the short-wavelength harmonic $\delta f_{e}$. In this case, the variation of the force acting on the photoelectron (cation) from the side of external and internal electric fields

$$
F_{j}=F_{j}^{\text {out }}\left(E_{j}^{\text {out }}\right)-n_{e p} \frac{\partial}{\partial x_{j}}\left[e E_{j}^{o w n}+\int e \varphi_{e p}\left(x_{j}-x_{j}^{\prime}\right) f_{e p}\left(x_{j}-x_{j}^{\prime}\right) \mathrm{d}^{3} x_{j}^{\prime}\right]
$$


where $p=d, s ; n_{e p}=\left\langle f_{e p}\right\rangle$ is the average density of plasma electrons; The $\varphi_{e p}$ is scalar potential of photoelectrons (cations) in the Coulomb field of plasma waves and the distributions of cations (photoelectrons) reflect either oscillations at the bottom of the corresponding potential valleys or tunneling through barriers when the threshold beam velocities $V_{e}^{t h r}$ are reached. Note that photoelectrons and cations under the influence of external fields move through a periodic sequence of potential barriers $+U(x)$ and wells $-U(x)$, while the photoelectron distribution is Boltzmann's: $\bar{f}_{\text {phe }} \sim n_{p h e} \exp \left(-\varepsilon / k T_{e}\right)$, where $\varepsilon=p_{x}^{2} / 2 m_{e} \pm U(x), U(x)=-e \varphi_{e}$. If we put $\omega_{r e p} \cdot \delta f_{e}$ on the right side of equation (16), and then multiply the left and right sides of (16) by the variation $\delta F_{x}$ and average in direct space over coordinates and time, then the correlator obtained at different times can be reduced to correlators (18), (19), (19'). In what follows, we restrict ourselves to the case of low temperatures, when such nonlinear effects as scattering by lattice vibrations and ionized impurities do not play a significant role [2]. Here, in $\delta$-collisions, the equilibrium distribution $f_{0}$ is displaced in the momentum space along the $p_{x}$ axis abruptly by $\left|P_{x}^{t h r}\right|$ both in the positive and negative directions of this axis without changing the shape $f_{0}(p)$. Suppose that, under the influence of an external uniform electric field $E^{o u t}$, the distribution of photoelectrons in the defect nucleus shifts in the positive direction of the $+p_{x}$ axis. According to (21), the second peak (protrusion) $\delta f_{e}(-)_{2}$ serves as a barrier to the passage of the drift current of these electrons. In turn, the cations in $\delta f_{e}(+)_{1}$ in one direction $+p_{x}$ are affected by the Coulomb attraction of the photoelectron distribution, which plays a decisive role, and the less significant Coulomb attraction of the hole distribution due to the small density, and also the field $E^{\text {out }}$ in the opposite direction. Here, the barrier for cations is the trough $f_{e}(-)_{1}$. In other words, when sliding, when the photoelectron velocity reaches the threshold values $V_{s l}^{t h r}>V_{g e n}^{\text {thr }}$, the ion plasma wave, consisting of a vacancy valley and two cationic protrusions, moves against the background of an additional potential relief created by the short-wavelength harmonic $\delta f_{e}$.

Let us consider the interaction of two dislocations $d 1$ and $d 2$ with arbitrary edge and screw components of the Burgers vector. In the framework of the plasma model, the long-wave propagating harmonics of plasma waves $d 1(d 2)$ interact with the oscillation currents $J_{\text {osc }}^{e}$ and rotations $J_{\text {turn }}^{e}$ in the additional potential relief of the dislocation core $d 2(d 1)$ created by short-wave harmonics that do not propagate in this core i.e. $J_{\text {osc }}^{e}, J_{\text {turn }}^{e}(d 1) \rightleftarrows J_{\text {osc }}^{e}, J_{\text {turn }}^{e}(d 2)$. The smoothness of electric fields from $J_{o s c}^{e}$ and magnetic fields from axial components of spiral currents from $d 1(d 2)$ to $d 2(d 1)$ core allows us to use quasiclassical dynamics in the form of Hamilton equations

$$
\begin{gathered}
\dot{\boldsymbol{r}}_{e}=\frac{\partial H_{s}}{\partial \boldsymbol{p}_{e}} \\
\dot{\boldsymbol{p}}_{e}=-\frac{\partial H_{s}}{\partial \boldsymbol{r}_{e}}
\end{gathered}
$$


where instead of the Hamiltonian of the system $H_{s}$ of two subsystems of pairs of photoelectrons and cations $d 1$ and $d 2$ we substitute the interaction Hamiltonian $H_{1}$ in the interaction representation [11]

$$
H_{1}=\int \sum_{\mu \nu} J_{\mu}^{v}\left(\boldsymbol{r}^{\prime}\right) A_{\mu}^{v}\left(\boldsymbol{r}-\boldsymbol{r}^{\prime}\right) \mathrm{d} \boldsymbol{r}^{\prime}, v=e, \text { cat } ; \mu=\text { osc, turn }
$$

where in our case the vector potential $A_{\mu}^{v}$ depends on the difference between the coordinates of the charged particles of the source $d 1(d 2)$ and the test charge $d 2(d 1)$, which transformes (25) into the convolution integral, maximally simplifying the description of the force interaction $d 1$ and $d 2$ both in direct and in the reverse $\boldsymbol{k}$-space by means of the Borel convolution theorem, transforming (25) upon its Fourier transform into the usual product of the Fourier components of currents. Here $H_{1}$ is directly proportional to the scalar product $\boldsymbol{k}_{1} \cdot \boldsymbol{k}_{2}$, where $\boldsymbol{k}_{1}$ and $\boldsymbol{k}_{2}$ are the wave vectors of charged particles by $d 1$ and $d 2$, and the nature of the interaction is determined by the law $\cos \alpha$. For $0<\alpha<\pi / 2 \quad d 1$ and $d 2$ are attracted, and for $\pi / 2<\alpha<\pi$ they repel. Note that in the equilibrium state $d 1$ and $d 2$ in $\boldsymbol{k}$-space, the right-hand side of (24) contains $\delta\left(\boldsymbol{k}_{1}+\boldsymbol{k}_{2}\right)$. When considering accumulations of dislocations, a statistical description is applicable using binary correlation functions of currents and stresses $\sigma_{k l}^{i f}, \sigma_{m n}^{e l}$ in the corresponding spaces.

Of particular interest is the experimental confirmation of the plasma model. In Figure 1, the AFM image of the source of Frank-Read dislocations located near the surface of a single-crystal $\mathrm{KCl}$ sample is shown. On the installation SPM Femtoscan, the change in the topography of the crystal surface under the influence of a pulsed magnetic field in the contact regime was studied by the method of scanning atomic force microscopy (AFM). Cantilevers Micromasch CSC-12 with a nominal tip radius of no more than $10 \mathrm{~nm}$ were used for scanning. Samples of a single-crystal $\mathrm{KCl}$ with dimensions $5 \times 5 \times 2 \mathrm{~mm}$ were obtained by cleavage along the cleavage planes, and were subjected to treatment with a weak pulsed magnetic field with induction $\mathrm{B}=0.12 \mathrm{~T}$ at room temperature in the time interval $\sim 30 \mathrm{~s}$. The maximum result of this action was found after the after effect time 48 hours and corresponded to the appearance of linear defects in the form of Frank-Read sources near the cleavage surface. To obtain an AFM image by atomic force microscopy, we used the contact quasistatic regime, where the tip of the probe was at a distance of $0.1-0.3 \mathrm{~nm}$ from the surface. Here, the source dislocation segment occupies a common field of view of $5 \times 10^{3} \mathrm{~nm} \times 5 \times 10^{3}$ $\mathrm{nm}$. AFM image includes three cross-sections of a common field: one central in the middle of the segment, two others near the attachment points. We give a qualitative analysis of the AFM image:

1) The surface relief on the AFM image consists of valleys and protrusions of various depths and widths. Cross-sections of the general field reflect the redistribution of the substance at the time of passage of the probe;

2) An insulated dislocation core, in the environment of which there are no linear (dislocations) and flat (interface, ledges, steps of terraces) defects, has a central trough relief with a depth of $4-6 \mathrm{~nm}$ and a width of $100-140 \mathrm{~nm}$, which 

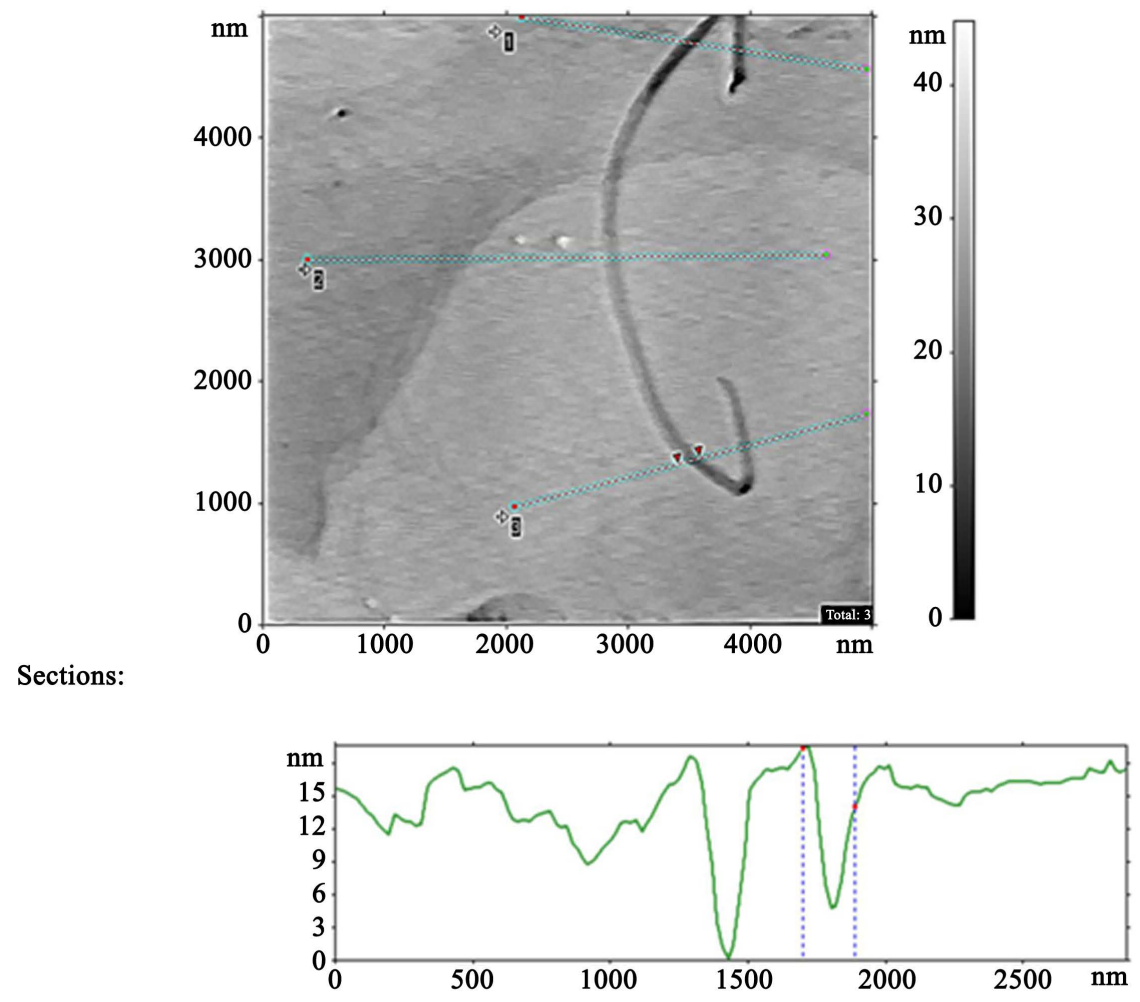

dX: $188.6 \mathrm{~nm}$ dY: $5.391 \mathrm{~nm}$

Step size: $5.391 \mathrm{~nm}$

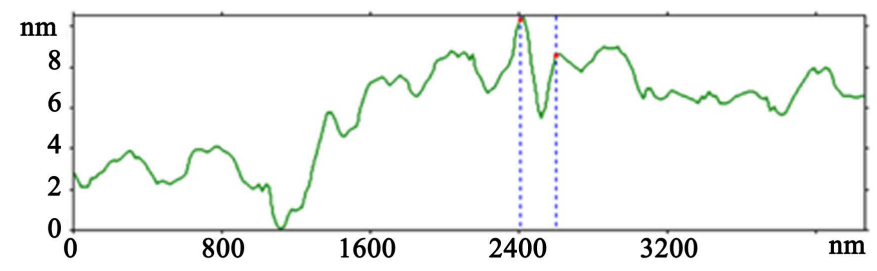

dX: $191.8 \mathrm{~nm}$ dY: $1.782 \mathrm{~nm}$

Step size: $1.782 \mathrm{~nm}$

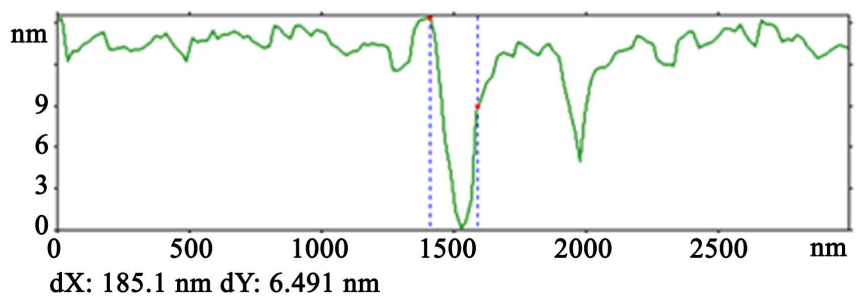

Step size: $2.362 \mathrm{~nm}$ and $6.491 \mathrm{~nm}$

Figure 1. AFM-image of the Frank-Reed source. The general field of view and three cross-sections: the central and two extreme near the fixation points.

corresponds to a vacancy valley, and two protrusions, the dislocation cores corresponding to the cationic protrusions are of approximately the same parameters, while the distribution of the protrusions and troughs of relief is symmetrical with respect to the axial plane passing through the minimum of the central relief trough (dislocation line) and has an approximately oscillatory character; a line approximately parallel to the abscissa axis, relative to which the sum of the 
areas above and below it is equal to zero, will be called the average level of the profile $\bar{L}_{p r}$;

3) The approach of a dislocation to a deep step leads to a decrease in $\bar{L}_{p r}$ from the dislocation line to this step and to an increase in the wavelength and profile amplitude in this interval;

4) The convergence of two dislocations of the same sign near the source fixation points leads to a significant deepening: up to $9-10 \mathrm{~nm}$, and expansion: up to $200-300 \mathrm{~nm}$, the troughs of the vacancy valleys of the nuclei of the dislocations, separated by a wide protrusion, while $\bar{L}_{p r}$ monotonically rises upward from both the left and on the right.

\section{Discussion of the Results}

1) Here a natural question arises: What is the reason for the appearance of the plasma model of linear defect nuclei? First, the impossibility of sufficiently reliably representing the essence and mechanisms of electroplastic and magnetoplastic effects [13]. Secondly, within the framework of the theory of atom-vacancy states, using the continuum model of concentration waves, it was shown that a dislocation is born as a solution of a nonlinear wave equation ([14], p. 23) without revealing the structure of the defect nucleus. Third, the well-known atomic models [15] are based on the consideration of displacement fields caused by the introduction or removal of excess atoms into the crystal lattice without taking into account the density of charged particles, and the discrete model of the Reed dislocation core [1] [2] in semiconductors does not allow displaying generation and slip defect nuclei without corresponding displacements of excess atoms. Fourth, over the past 30 years, a number of new experimental methods have been created and tested, including atomic force microscopy, which allow us to take a fresh look at the problem of generation and slip of linear defect nuclei. It is clear that for a reliable experimental substantiation of the plasma model, a sufficiently large data set will be required.

2) There is a fundamental difference between the Reed model and the plasma model. In the Reed model, conduction electrons captured by unsaturated bonds of a chain of atoms along the edge of the half-plane inserted into the crystal form a linear charge. In the plasma model, as a result of the internal photoelectric effect, pairs of cations and valence electrons knocked out from semiconductor atoms are formed, as well as pairs of cations of alkali halide crystals, other dielectrics and bond electrons under the influence of an alternating field. In this case, the distribution of conduction electrons forms short-wave non-propagating harmonics of plasma waves, forming the structure of the defect nucleus and, at the same time, an additional potential relief of the crystal in which the distribution of the above pairs moves.

\section{Conflicts of Interest}

The author declares no conflicts of interest regarding the publication of this paper. 


\section{References}

[1] Matare, H.F. (1971) Electronics of Defects in Semiconductors. Wiley Interscience. A Division of J. Wiley and Sons, Inc. New York, London, Sydney, Toronto.

[2] Seeger, K.H. (1973) Semiconductors Physics. Springer-Verlag, Wien, New York.

[3] Vershinin, Yu.N., Il'ichev, D.S. and Morozov, P.A. (2000) The Effect of Shock Compression of Solid Dielectrics on the Injection of Valence Electrons in Strong Electric Fields. Journal of Technical Physics, 70, 84-87. https://doi.org/10.1134/1.1259575

[4] Fever, P. (1952) Electronic States in Crystals under Large Over-All Perturbations. Journal Physic Review, 88, 92-101. https://journals.aps.org/pr/pdf/10.1103/PhysRev.88.92

[5] Keldysh, L.V. (1958) Behavior of Non-Metallic Crystals in Strong Electric Fields. Journal of Experimental and Technical Physics, 33, 994-1003. http://www.jetp.ac.ru/cgi-bin/dn/e_006_04_0763.pdf

[6] Reims, S. (1972) Theory of Many-Electron Systems. North-Holland Publishing Company, Amsterdam, London.

[7] Gorshkov, B.G. (1982) Investigation of the Mechanisms of Destruction of Ionic Crystals under the Action of Pulsed Laser Radiation of the Nanosecond Range. Transactions of Phys. Inst. Acad. Nauk, 136, 81-136.

[8] Kazantsev, S.G. (2003) Laser Resistance of Promising Materials of IR-Optics. Mechanisms of Damage to Materials of Optical Elements of Power Optics. Optics of the Atmosphere and Ocean, 6, 390-401.

[9] Lifshits, E.M. and Pitaevsky, L.P. (1979) Physical Kinetics. Nauka, Moscow.

[10] Kittel, C. (1976) Introduction to Solid State Physics. 4th Edition, J. Wiley and Sons, New York, London, Sydney, Toronto.

[11] Busov, V.L. (2019) Dynamic Equations of Evolution in the Nuclei of Linear Defects of Crystalline Materials during the Collision of Solids. PhysicalMesomechanic, 22, 91-96.

[12] Landau, L.D. and Lifshits, E.M. (1973) Mechanics. Nauka, Moscow.

[13] Golovin, Yu.I. (2004) Magnetoplasticity of Solids (Review). Journal of Solid State Physics, 46, 769-803. https://doi.org/10.1134/1.1744954

[14] Panin, V.E., Likhachev, V.A. and Grinyaev, Yu.V. (1985) Structural Levels of Deformation of Solids. Nauka, Siberian Branch, Novosibirsk.

[15] Mirkin, L.I. (1968) The Physical Basis of Strength and Plastisity. Publishing House of Moscow State University, Moscow. 